\section{Bernhard Uehleke}

Berlin, Deutschland

\title{
Professor Dr. Dr. h.c. Heinz Schilcher - † 17.06.2015
}

er Generationen von Ärzten und fungierte langjährig als Vorstandsmitglied des Zentralverbands der Ärzte für Naturheilverfahren und Regulationsmedizin e.V. (ZAEN). Später entwickelte er das Konzept der Weiterbildung in Phytotherapie für Apotheker.

Schon vor und während dem Studium interessierte Schilcher sich zusätzlich für die Medizin - einem Praktikum in der Krankenhausapotheke der Barmherzigen Brüder in Neuburg schloss er gleich ein medizinisches Praktikum an. Von 1952 bis 1956 studierte er Pharmazie an der LudwigMaximilians-Universität in München und promovierte 1959 bei Ludwig Hörhammer zum Thema «Zur Kenntnis der Inhaltsstoffe von Lycopus europaeus L. und Lycopus virginicus L. (Wolfstrappkraut) und deren Wirkungen bei Hyperthyreose» am Institut für Pharmazeutische Arzneimittellehre. Er führte chromatographische Analysen durch und prüfte antigonadotrope Effekte an Kaulquappen.

Als Leiter der Wissenschaftlichen Abteilung sowie Herstellungs- und Kontrollleiter der Firma Salus entwickelte Schilcher von 1963 bis 1974 etliche Phytopharmaka und führte dabei pharmakologische und klinische Prüfungen durch. Er schlug 1964 eine Standardisierung für Phytopharmaka anhand von chromatographischen Fingerprints, ergänzt durch physikalische Messparameter, vor. Später schuf er in zahlreichen Publikationen die theoretischen Grundlagen für eine analytische Charakterisierung von pflanzlichen Zubereitungen; so hat er Begriffe wie «Koeffektoren», «Leitsubstanzen» und «wirksamkeitsmitbestimmende Inhaltsstoffe» geprägt.

Nach seiner Tätigkeit an der Universität Marburg bei Professor Wichtl ging Schilcher als Forschungsleiter zur Firma Fink und unterrichtete nebenbei in Tübingen, wo er bei Professor Ernst seine Habilitationsschrift über die Kamille verfasste. Von 1983 bis 1995 war er dann Professor an der Freien Universität Berlin und amtierte zeitweilig auch als Dekan. In dieser Zeit und danach war Schilcher in der Kommission E insgesamt über 25 Jahre tonangebend und brachte dort viele Ideen ein. Dabei ging er niemals einer Auseinandersetzung aus dem Weg schliesslich war er doch meistens besser informiert und vorbereitet als die anderen. Die meisten Monographien tragen somit seine Handschrift. Er begründete massgeblich auch das Konzept der traditionellen Phytopharmaka und arbeitete sehr aktiv an der entsprechenden Indikationsliste mit.

Durch seine zahlreichen Buchbeiträge und Monographien bleibt Schilcher einer der bedeutendsten Protagonisten der modernen Phytotherapie. Sein Fachbuch «Leitfaden Phytotherapie», das er mit Susanne Kammerer im Urban \& Fischer Verlag im Jahre 2000 veröffentlichte, ist inzwischen unter der Herausgeberschaft von Schilcher, Kammerer und Wegener in vierter Auflage mit 1200 Seiten erschienen. genannte Zusatzbezeichnung schulte

\section{KARGER}

(c) 2015 S. Karger GmbH, Freiburg

Fax +497614520714 
Dieses Buch bezeichnet Schilcher selbst als sein wichtigstes Lebenswerk betreffend der Bezüge einer Heilpflanze, ihrer Qualität sowie ihrer Verarbeitung zur Anwendung in der ärztlichen Praxis.

Aus seiner ersten Ehe entstammt der Sohn Stefan. Seine Frau Barbara ist ebenfalls Apothekerin und unterstützte Schilcher bei seinen Arbeiten, Reisen und Hobbys. Zu Letzteren gehörten vor allem Wildwasser-Kanu-Fahren und Skifahren - beide Sportarten übte er bis in das höchste Alter aktiv aus.

Schilcher erhielt für sein Engagement und seine Arbeiten, Beiträge, Fachpublikationen und Fachbücher zahlreiche Auszeichnungen - vom Sebastian-Kneipp-Preis bis hin zu seinen beiden Ehrendoktorwürden:

- 1985: Sebastian-Kneipp-Preis,

- 1988: Rudolf-Fritz-Weiss-Preis,

- 1990: Universitäts-Verdienstmedaille der Semmelweis-Universität Budapest, Ungarn,

- 1991: Sertürner-Gedenkmünze,

- 1993: Bundesverdienstkreuz 1. Klasse des Verdienstordens der Bundesrepublik Deutschland,

- 1994: Aulus-Cornelius-Celsus-Medaille des Zentralverbands der Ärzte für Naturheilverfahren,

- 1997: Umweltpreis der Bayerischen Staatsregierung,

- 2002-2007: Ehrenmitglied der Gesellschaft für Arzneipflanzenfor- schung (GA), der Gesellschaft für Phytotherapie, des Instituts für Heilpflanzenforschung in Poznan (Posen) und der Ungarischen Gesellschaft für Phytotherapie,

- 2003: Ehrendoktorwürde der Universität für Medizin und Pharmazie Tirgu-Mures (Neumarkt/Siebenbürgen),

- 2003: Ehrenpräsident des Zentralverbands der Ärzte für Naturheilverfahren,

- 2006: 20th Century Authority Award for Chamomile Research and Development,

- 2007: Ehrendoktorwürde der Semmelweis-Universität Budapest, Ungarn,

- 2009: Ehrenpreis der Gemeinnützigen Forschungsvereinigung Saluplanta e.V.

Für die Zukunft forderte Professor Schilcher ein Öffnen der Wissenschaft «aus dem Elfenbeinturm», einen besseren Dialog zwischen Apothekern und Ärzten sowie auch mehr Kommunikation mit nichtakademischen Kräuterexperten. Letztere sollten sich aber auch stets über den Stand der Wissenschaft informieren. Sein Ziel war es, das Wissen über die Botanik und die traditionelle Anwendung bzw. Erfahrung auf der einen Seite mit der naturwissenschaftlichen und medizinischen Expertise auf der anderen Seite zu verschmelzen. An diesen Wunschvorstellungen, wie er selbst sie nannte, wollen wir in seinem Sinne weiterarbeiten ...

Eine kleine Episode: Ende der 1990er-Jahre hatten wir im Kurs «Heilkräuter-Experte» beim KneippBund in Bad Wörishofen gelehrt und hörten abends einem Seminar zu, das von dem Management-Trainer Peter Ebeling gehalten wurde. Dies war eben typisch der an allem interessierte Schilcher: Als perfekter Redner benötigte er mitnichten eine Nachhilfe in Rhetorik, und jeder andere hätte sich nach getaner Arbeit zurückgezogen oder etwa an der Bar eingefunden ... Ebeling empfahl unter anderem die sofortige Umsetzung von Vorhaben und Plänen und teilte zur Demonstration der begrenzten Zeit für das Aufschieben Papiermassbänder aus. Auf der $100 \mathrm{~cm}$ langen Skala schnitten wir zunächst unsere Lebensjahre ab. Von dem Rest bis 100 war dann die Lebenserwartung für die Männer bei 73 abzuschneiden. Heinz Schilcher schaute betroffen auf den nur fingerlangen «Rest» und gab seiner Hoffnung Ausdruck, dass es bei ihm doch etwas länger dauern möge. Angesichts seiner Fitness und seines gesunden Lebensstils schien eine solche Hoffnung durchaus berechtigt - und heute sind wir dankbar, dass Schilcher dann rund das Vierfache der damals zu erwartenden Zeitspanne erreichte und bis ins hohe Alter sehr aktiv war! 